\title{
Simulation of circular jet outfalls using artificial neural network
}

\author{
Seyed Habib Musavi-Jahromi and Javad Ahadiyan \\ Faculty of Water Sciences Engineering, Shahid Chamran University (SCU), Ahwaz, Iran.
}

Accepted 04 May, 2012

\begin{abstract}
In the present article, outfall of the circular jet in the stagnant ambient fluid has been investigated. First, dimensional analysis for eleven involved parameters in the process, results in four dimensionless numbers including; relative length of jet trajectory, $x / d p$; jet convergence angel, $\theta_{c}$; geometry number of jet, $\mathrm{Di} / \mathrm{dp}$ and Densimetric Froude Number, $\mathrm{Fr}_{\mathrm{d}}$. Furthermore, using an experimental setup in the hydraulic laboratory of Shahid Chamran University (SCU), a large amount of data was gathered from several runs of physical model. Gathered data include coordinates of trajectory $(x, z)$ and other involved variables. Then Qnet2000 as an artificial neural network (ANN) software was selected for simulation of the jet outfalls. In this regard, 192 runs of Qnet2000 were performed for training and testing processes. Then, in the application step, ANN model was used for predicting two values of $z$ as upper and inferior limits of the jet trajectory. Findings showed that the model could simulate upper and inferior limits of trajectory very successfully.
\end{abstract}

Key words: Submerged Jet, outfalls, physical modeling, artificial neural network.

\section{INTRODUCTION}

The present article undertakes an artificial intelligence approach for predicting hydraulic properties of circular buoyant jets in the static ambient flow. In many cases, due to high concentrations of pollutants, or critical toxicity or even very high temperature wastewaters from nuclear reactors, inevitably dilution of wastewater to reach the limit concentration should be done in shortest time. One way to make quick dilution is using submerged jets in rivers or seas that can relatively in short period dilute large amounts of pollution due to its mixture of high turbulence conditions, and the destructive effects will rapidly reduce (Fischer et al., 1979). Jet behaviors are very important in the environmental field and real ambient flow. Environmental regulations and water quality objectives are mostly set in terms of pollutant concentrations in receiving waters. It is important to be able to predict the concentration distribution in the vicinity (the near field) of a discharge for a given discharge design and location, waste load, and environmental conditions. This is required for defining mixing zones and minimizing the impact of discharges on sensitive receivers (e.g. nearby beaches for amenities, wetland reserve, or fisheries). The proper design of a submarine outfall can have a profound effect on the water quality actually observed near the discharge (Lee and Chu, 2003).

Turner (1967) and Kunze (1987) have investigated the salt fingering derived from a downward salinity flux. Maxworthy (1983) and Turner (1998) have shown that a two-dimensional surface intrusion, with a low Reynolds Number (R), will actually terminate its surface progression at the same distance and plunge downward. Cuthberston et al. (2008) studied particle deposition process for the case of a round, turbulent, particle-laden, buoyant jet, discharging horizontally into homogeneous receiving fluid that is initially either quiescent or coflowing. Ahadiyan and Musavi-Jahromi (2008) have investigated variation of efflux momentum in shallow receiving water, using FLOW-3D, and have shown that 
the momentum flux decreases in the longitudinal distance of jet position, which is agreed with Turner (1967), Kunze (1987), Maxworthy (1983), Turner (1998) and Cuthberston et al. (2008). Vertical round buoyant jets in a cross flow have been investigated by Ben et al. (2004) and Papanicolau and List (1988).

In the present article, outfall of the circular jet in the stagnant ambient fluid is going to be investigated using an experimental set up and artificial neural network, which has not been considered in the literature yet.

\section{Fundamentals}

Buoyant jet is a kind of a free turbulence flow (Albertson et al., 1950; Rajaratnam, 1976). French (1986), illustrated hydraulic characteristics of buoyant surface jets and associated phenomena. In this kind of flow, the velocity of the turbulence is proportional to the product of the mixing length and the mean velocity gradient.

If the Reynolds number for fluid efflux, from a submerged boundary outlet is not too low, the mean velocity, $v$, at any point should depend only on the coordinates $x, y$ and $z$ on the efflux velocity $v_{0}$ and on a linear dimension, $\mathrm{L}_{0}$. Thus, dimensionless relationships between these parameters are:

$$
\frac{v}{v_{0}}=f_{l}\left(\frac{x}{L_{0}}, \frac{y}{x}, \frac{z}{x}\right)
$$

Equation 1 is considered to involve the magnitude of $v$ and the components of which may be related through the differential continuity equation. The flux of flow, $Q$, past successive normal sections, may be written as the integral of the differential flux $\mathrm{v}_{\mathrm{x}}{ }^{*} \mathrm{dA}$ over any normal section. Since the entrainment, $Q$ will vary with the longitudinal distance $x$ from the efflux section, its ratio to the efflux rate $Q_{0}$ is as follows:

$$
\frac{Q}{Q_{0}}=\frac{\int_{0}^{\infty} v_{x} d A}{v_{0} A_{0}}=f_{2}\left(\frac{x}{L_{0}}\right)
$$

In which $A_{0}$ is the cross sectional area of the outlet. Similarly, since the momentum flux $M$ may be written as the integral of the volume flux $v_{x}{ }^{*} \mathrm{~d} A$, the longitudinal component of momentum per unit volume $\rho \mathrm{v}_{\mathrm{x}}, \rho$ being the fluid density, the ratio of $M$ for any section to $M_{0}$ for the efflux section should be:

$$
\frac{M}{M_{0}}=\frac{\int_{0}^{\infty}\left(v_{x}\right)^{2} d A}{\left(v_{0}\right)^{2} A_{0}}=f_{3}\left(\frac{x}{L_{0}}\right)
$$

The hydrostatic force against jet flow causes the deceleration of the jet and the acceleration of the surrounding fluid is the tangential shear within the mixing region; because this process is wholly internal, it follows at once that the momentum flux must be a constant for all normal sections of a given flow pattern:

$$
\frac{M}{M_{0}}=\frac{\int_{0}^{\infty}\left(v_{x}\right)^{2} d A}{\left(v_{0}\right)^{2} A_{0}}=1
$$

If, moreover, viscous action is presumed to have no influence on the mixing process, the diffusion characteristics, and hence the characteristics of the mean flow should be dynamically similar under every condition. In this research, predicting the hydraulic parameters and geometry of the trajectory in the buoyant round jets, including the upper and lower limits and length as shown in Figure 1, are investigated using physical modeling and ANN and artificial neural network.

Learning approach is appealing for artificial intelligence, since it is based on the principle of learning from training and experience. ANNs are well suited for learning, where connection weights are adjusted to improve the performance of a network. An ANN is a network of neurons, connected with directed arcs, each with a numerical weight, specifying the strength of the connection. These weights indicate the influence of previous neuron on the next neuron where positive weights represent reinforcement and negative weights represent inhibition (Gallant, 1993). Generally, the initial connection weights are random.

\section{MATERIALS AND METHODS}

\section{Dimensional analysis}

According to jet governing parameters, to achieve non-dimensional equation, dimensional analysis has been performed. The variables involved in the jet hydraulic are as follows:

$$
f\left(\rho_{a}, \mu_{j}, u_{0}, d_{p}, D_{i}, x, \rho_{j}, g, Z_{u}, Z_{2}, \theta_{c}\right)=0
$$

Where $\rho_{a}$, is ambient density; $\mu_{j}$, is viscosity of jet injection; $u_{0}$, is efflux velocity; $\mathrm{dp}$, is port diameter; $\mathrm{Di}$, inlet diameter; $\mathrm{x}$, length from jet location; $\rho_{j}$, jet injection density; $\mathrm{g}$, acceleration due to gravity; $Z_{\mathrm{u}}$, height of trajectory upper limit; $Z_{2}$, height of trajectory inferior limit and $\theta_{c}$, is convergence angle of jet nozzle. Finally, using dimensional analysis, the non-dimensional parameters were derived as follow:

$$
f\left(\frac{\rho_{j} \cdot U_{0} \cdot d_{p}}{\mu_{j}}, \frac{U_{0}}{\sqrt{\left(\frac{\Delta \rho}{\rho_{a}} \cdot g \cdot d_{p}\right)}}, \frac{Z_{u}}{d_{p}}, \frac{Z_{2}}{d_{p}}, \frac{x}{d_{p}} \frac{D_{i}}{d_{p}}, \theta_{c}\right)=0
$$




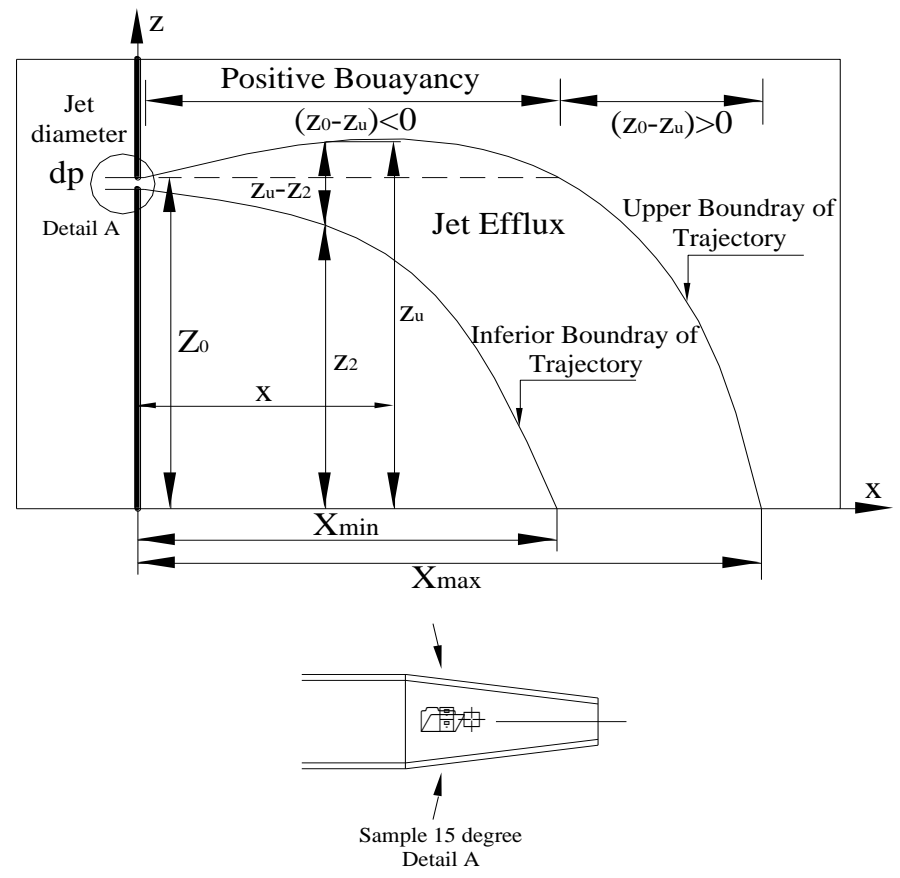

Figure 1. Schematic representation of the jet outfall and its nozzle details.

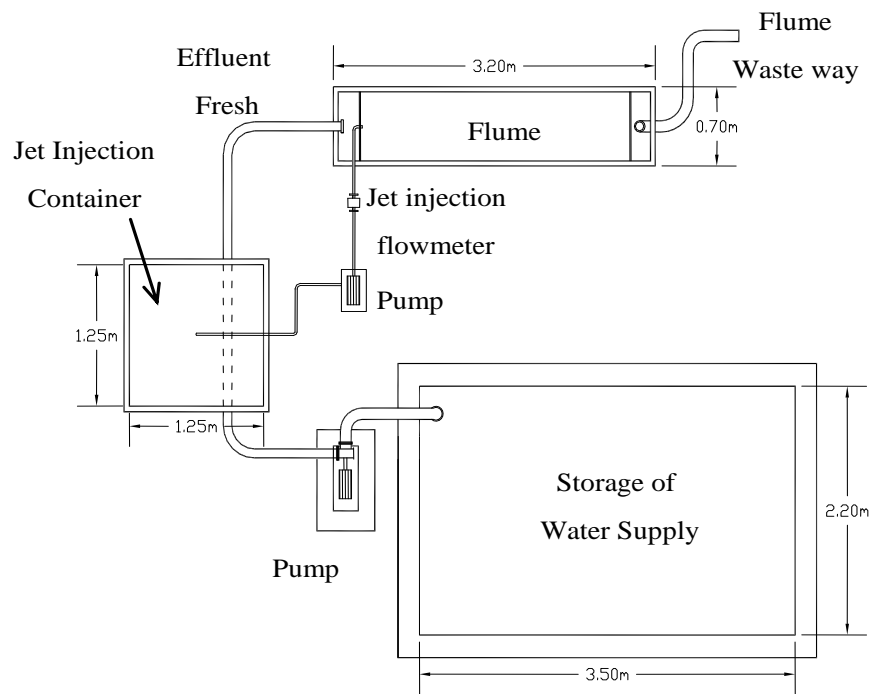

Figure 2. Schematic of the experimental setup.

Where in equation (6) the first parameter, is Reynolds number of jet efflux, the second parameter is Densimetric Froude Number [dimensional analysis gives geometric and hydraulic characteristics of the jet flow in the static ambient fluid as function of Densimetric Froude Number (Adrian and Stephan, 2004; Pantokratoras, 2003)], the third, fourth and fifth parameters denote coordinate of trajectory, the sixth parameter is the geometric number of jet and the last parameter is the convergence angle. In this research, trajectories geometry of round jet, which they had measured with artificial neural network, was predicted. The variables include various discharges, concentration, and diameter.

The data were gathered, using a constructed physical model, with $3.2 \mathrm{~m}$ length, $0.7 \mathrm{~m}$ width and $0.95 \mathrm{~m}$ height, in the hydraulic laboratory, Shahid Chamran University, Ahwaz, (SCU), Iran (2007 to 2008). The schematic representation of flume and its equipments are shown in Figure 2. This figure represents supply of fresh water from concrete storage tank, using a pump with $20 \mathrm{~L} / \mathrm{s}$ and $15 \mathrm{~m}$ of head. The means of supply pump, experimental flume from fresh water is to be filled. Jet injection through salt density current, is also transferred into experimental flume. Measurements include both 


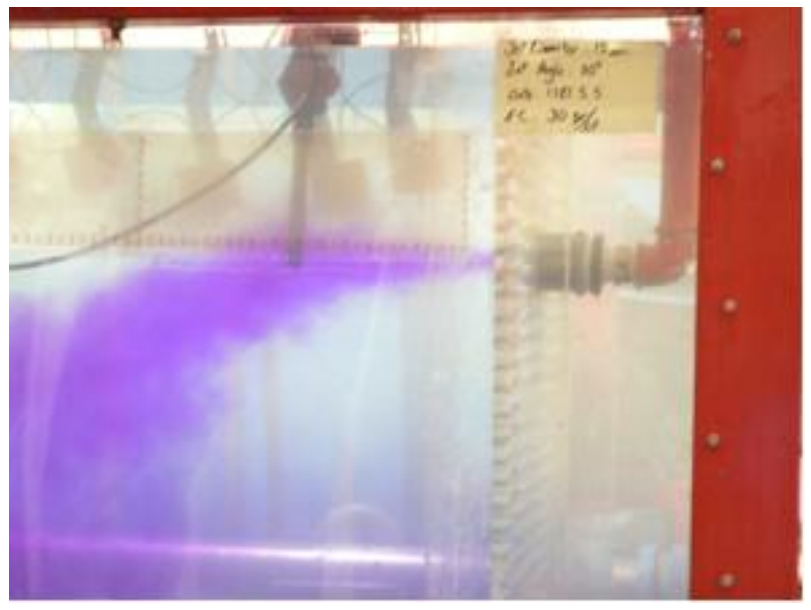

Figure 3. observed trajectory in the stagnant ambient flow in the hydraulic laboratory.

Table 1. Designed experimental scenarios of the research program.

\begin{tabular}{cclc}
\hline Concentration $\mathbf{( g / L )}$ & Diameter $(\mathbf{m m})$ & Discharge L/s & Convergence angle $\left.\mathbf{(}^{\circ}\right)$ \\
\hline 15 & & & 15 \\
30 & 5 & 4 cases different & 30 \\
50 & 8 & efflux velocity & 45 \\
200 & 15 & & 60 \\
& & & 90 \\
\hline
\end{tabular}

length and height of trajectory under different jet injection concentrations and constant temperature of jet injection. Figure 3 shows the real trajectory in the stagnant ambient flow, developed in the hydraulic laboratory.

As observed in the Figure 3, there are two boundaries for trajectory; one as upper limit of trajectory, and another one as inferior limit. The data gathered include results of experiments which had been performed in three diameters, including 5, 8 and 15 $\mathrm{mm}$ of round jet and 4 discharges. In the entire experimental data, thermal condition between jet injection and ambient fluid was constant. In performed experiments, the fluid density was measured, using a hydrometer $151 \mathrm{H}$ standardized carried out with ASTM E100 (2003) method, respectively. As shown in Table 1, several scenarios are considered to be investigated.

\section{Artificial neural network}

Trained ANNs can be used for prediction of outputs of new unknown patterns (Adineh et al., 2008). The advantages of using of ANNs are; high computation rate, learning ability through pattern presentation, prediction of unknown pattern, and flexibility affronts the noisy patterns (Hagan and Menhaj, 1994). In this research, feed forward networks are used. In addition, Levenberg-Marquardt (LM) regulation learning algorithms was utilized. The network types include:

\section{Feed-forward back-propagation (FFBP)}

This network consists of one input layer, one or several hidden layers and one output layer. Usually, back propagation (BP) learning algorithm is used to train this network (which finally result in learning). In the case of BP algorithm, first, the output layer weights are updated. For each neuron of the output layer, a desired value exists. By this value and the learning rules, weight coefficient is updated. For some problems, the BP algorithm presents suitable results, while it ends to improper results for the others. In some cases, the learning process is upset because of trapping in local minimum. This is because of the answer lying at the smooth part of the threshold function. Figure 4 shows the training process of $\mathrm{BP}$ algorithm for the updating weights and biases. During the training of this network, calculations were performed from input of network toward the output, and then the values of errors were propagated to prior layers. Calculations were done layer-to-layer and the output of each layer was the input of next layer.

\section{Cascade-forward back-propagation (CFBP)}

This network like FFBP network uses the BP algorithm for updating weights, but the main symptom of this network is that each layer's neurons are related to all the previous layer neurons. Two training algorithms are used for updating the network weights. These algorithms are LM and Bayesian regulation BP algorithms.

For the LM algorithm, gradient-based training algorithms, such as $\mathrm{BP}$, are the most commonly used by researchers. They are not efficient because the gradient vanishes at the solution. Hessianbased algorithms allow the network to learn features of a complicated mapping more suitably. The training process converges quickly as the solution is approached, because the Hessian does not vanish at the solution.

To benefit from the advantages of Hessian-based training, LM algorithm is used. The LM algorithm is a Hessian-based algorithm 


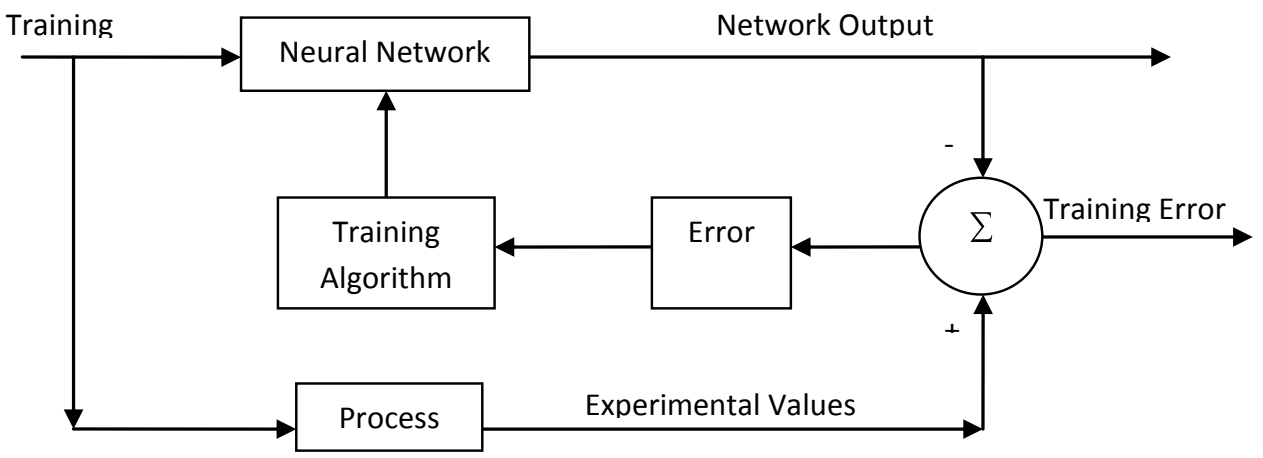

Figure 4. Training process of the back propagation networks.

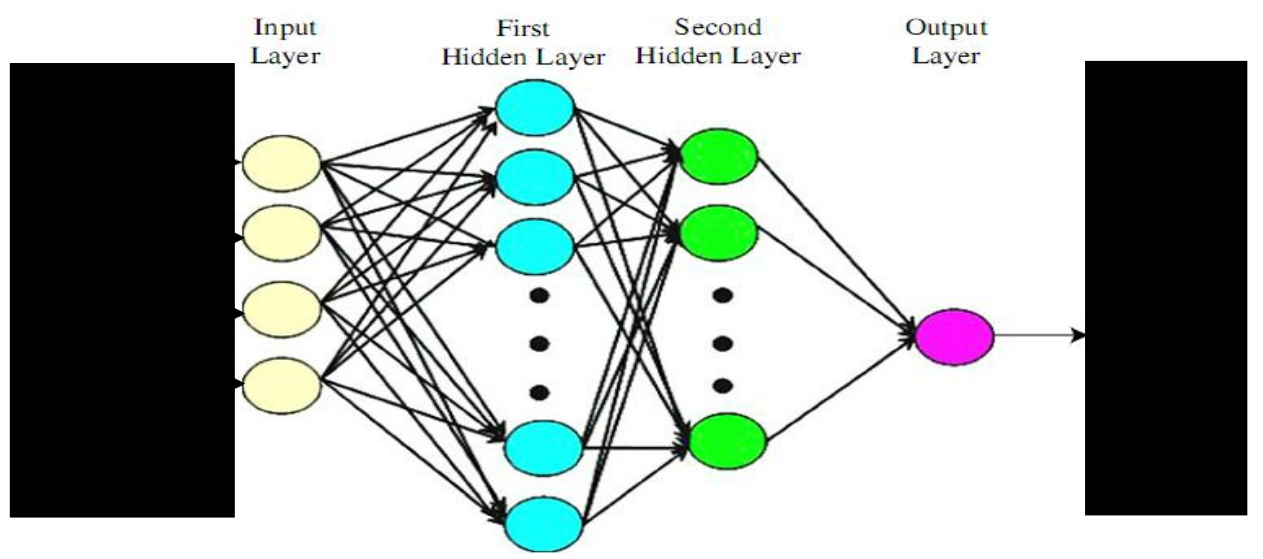

Figure 5. Applied ANN's topology.

for non-linear least squares optimization. The steps involved in training a neural network in batch mode, using LM algorithm, are as follows (Girosi et al., 1995):

1. Introduce all inputs to the network and computing the corresponding network outputs and errors;

2. Computation of the Jacobian matrix J;

3. Solution of LM weight updates equation as follows:

$x_{k+1}=x_{k}-\left[J^{T} J+\mu I\right]^{-1} J^{T} e$

where, $x_{k}$ is calculated weight in prior step; $x$, is weight value in new step; $\mu$, is training parameter; $J_{k+1}^{\top} J$, is Hessian matrix; I is the identity matrix; and $\mathrm{J}^{\top} \mathrm{e}$, is the gradient (where e is the network error vector).

4. Error computation using $x+\Delta x$. If this new error is smaller than that computed in step 1 , then reduce the training parameter $i^{\prime}$ by $\mu$ and $\mu^{+}$; let $x=x+\Delta x, \mu^{-}$and $\mu^{+}$are predefined values set by the user.

In the Bayesian regularization (BR) algorithm, training process of $\mathrm{BP}$, with $\mathrm{BR}$ algorithm, is initialized with random distribution of initial weights and biases. After presenting input patterns to networks, updating initial weight begins to obtain final distribution using algorithm. This procedure has a good approximation for training step, and can improve generalization performance. In this algorithm, instead of the sum of squared error (SSE) on the training set, a cost function, which is the SSE plus a penalty term, is automatically adjusted (Kozma et al., 1996).
Structural learning with forgetting is the main technique used for regularization (Kozma et al., 1996; Pirmoradian et al., 2009). It has good approximation with arbitrary accuracy for training, and it can improve generalization performance. The BR algorithm foundations are:

1. The performance function used for optimization is as follows:

$J=S S E+\lambda \sum w^{2}$

Where $\lambda$, is penalty factor within the range $[0,1]$ and $\sum w^{2}$ is the sum of square weights (SSW);

2. Network architecture includes selection of hidden layer and threshold function;

3. The prior distribution is setup with Gaussian distribution. Sensitivity analysis is performed in order to decrease the chance of being trapped in a local minimum and to find stable results.

Applying four inputs in all experiments, the output was derived from different situation. The following conditions are considered; networks with four neurons in input layer (relative length of jet trajectory, $X / d p$, which $X$ is length of positive buoyancy and $d p$ is port diameter; jet convergence angel $\theta_{c}$; geometry number of jet $\mathrm{Di} / \mathrm{dp}$; Densimetric Froude Number $\mathrm{Fr}_{d}$; and one neuron in output layer (relative height of jet trajectory $\mathrm{Z} / \mathrm{dp}$ ) were designed. Figure 5 shows the considered neural network topology, input, and output parameters. 
Table 2. Maximum and minimum of parameters before and after normalization.

\begin{tabular}{lccccc}
\hline & $\mathbf{x} / \mathbf{d p}$ & $\boldsymbol{\Theta}_{\mathbf{c}}$ & $\mathrm{Di} / \mathbf{d p}$ & $\mathbf{F r}_{\mathbf{d}}$ & $\mathbf{z} / \mathbf{d p}$ \\
\hline \multicolumn{4}{l}{ Before } \\
Min & 0 & 15 & 2.54 & 7.28 & 0 \\
Max & 414 & 90 & 5.08 & 123.41 & 130 \\
\multicolumn{7}{l}{ After normalization } \\
Min & 0 & 0 & 0 & 0 & 0 \\
Max & 1 & 1 & 1 & 1 & 1 \\
\hline
\end{tabular}

Qnet2000 ${ }^{\odot}$ software is used for this research. The Qnet2000 ${ }^{\odot}$ provides a complete set of functions and a graphical user interface for the design, implementation, visualization, and simulation of neural networks. It supports the most commonly used supervised and unsupervised network architectures and a comprehensive set of training and learning functions (Demuth et al., 2007).

For obtaining the desired answer, FFBP networks was utilized. Training process by this network is repetitive, when the error between desired value and predicted value is minimum and the training process moves towards stability. The increasing method was used for selection layers and neurons for evaluation of various topologies. By this method, when the network traps into the local minimum, new neurons are added to network gradually. This method has the more practical potential for detecting the optimum size of network. The advantages of this method is that network complexity increases gradually by increase in neurons. The optimum size of network is always obtained by adjustments. Monitoring and evaluating local minimum is done during the training process (Kozma et al., 1996). Various threshold functions were used to obtain the optimized status (Kasabov, 1998).

$$
\begin{aligned}
Y_{j} & =\frac{1}{1+\exp \left(-X_{j}\right)}(\text { LOGSIG }) \\
Y_{J} & =\frac{2}{\left(1+\exp \left(-2 X_{j}\right)\right)-1}(\text { TANSIG })
\end{aligned}
$$

$\mathrm{Xj}$, is sum of weighted inputs for each neuron in the $\mathrm{jth}$ layer and can be computed using

$$
X_{j}=\sum_{i=1}^{m} W_{i j} * Y_{i}+b_{j}
$$

Where $\mathrm{m}$, is number of output layer neurons; $\mathrm{W}_{\mathrm{ij}}$, is the weight between $i^{\text {th }}$ and $j^{\text {th }}$ layers; $Y_{i}$, is the $i^{\text {th }}$ neuron output; and $b$, is the bias of the $j^{\text {th }}$ neuron for FFBP network. Experimental values for up and down boundaries are 1709 and 1514 patterns, respectively. About $80 \%$ of all data are random for training of network with suitable topology and training algorithms. Data for training step entered to the network, and the network is adjusted according to its error. The remaining data were used for test. Testing data have no effect on training, and provide an independent measure of network performance, during and after the training.

The performance of the neural network model was evaluated, using the root mean square error (RMSE) and coefficient of determination $\left(R^{2}\right)$, between predicted and measur ed values of geometric and hydraulic properties. When the RMSE value is minimum, and $R^{2}$ is $\geq 0.8$, a model can be judged as very good. The RMSE can be calculated by the below-mentioned equation, where $T$ denotes the number of data patterns; $S$, is network output of $k^{\text {th }}$ pattern and $T_{k}$, is target output of $k^{\text {th }}$ pattern (observed data).

$R M S E=\sqrt{\frac{1}{T} \sum_{K=1}^{T}\left(S_{k}-T_{k}\right)^{2}}$

In addition, the coefficient of determination is

$$
\begin{aligned}
& R^{2}=1-\frac{\sum_{k=1}^{T}\left[S_{k}-T_{k}\right]}{\sum_{k=1}^{T}\left[S_{k}-T_{m}\right]} \\
& \text { with } T_{m}=\frac{\sum_{k=1}^{T} S_{k}}{T}
\end{aligned}
$$

Another criterion is the mean absolute error, which is calculated from the following equation:

$$
M A E=\frac{1}{T} \sum_{k=1}^{T}\left|S_{k}-T_{k}\right|
$$

For increasing the accuracy and processing velocity of network, the input and output data were normalized and de-normalized, before and after the actual application in the network. Input data were normalized at a boundary of $[0,1]$ using:

$$
X_{n}=\frac{X_{i}-X_{\min }}{X_{\max }-X_{\min }}
$$

Where $X_{n}$, is normalized value; $X_{i}$, is real value; $X_{\text {min }}$, is minimum real value and; $X_{\max }$, is maximum real value. The maximum and minimum of parameters are shown in Table 2. Qnet2000 ${ }^{\odot}$ is capable of normalizing input and output data automatically.

\section{RESULTS}

Jet trajectory has two boundaries, upper and inferior. Using original experimental setup in the hydraulic laboratory of Shahid Chamran University (SCU), Iran, several upper and inferior limits were observed. Longitudinal 2D measured trajectory profiles are shown in Figure 6 for $1.55 \mathrm{~m} / \mathrm{s}$ jet flow velocity. The coordinate of limit boundary of trajectory are $x$ and $z$. Due to symmetry towards lateral direction, no y was measured. As shown in Figure 6, the length of the trajectory, upper and lower limits are increased when $\theta_{c}$ is increased. As shown in Figure $6 \mathrm{a}$, however, the length of the lower limit of the trajectory for $\theta_{c}=15^{\circ}$ is $1080 \mathrm{~mm}$, it is $1400 \mathrm{~mm} \mathrm{(30 \%}$ increase) for $\theta_{c}=90^{\circ}$ (Figure 6d). Almost the same 

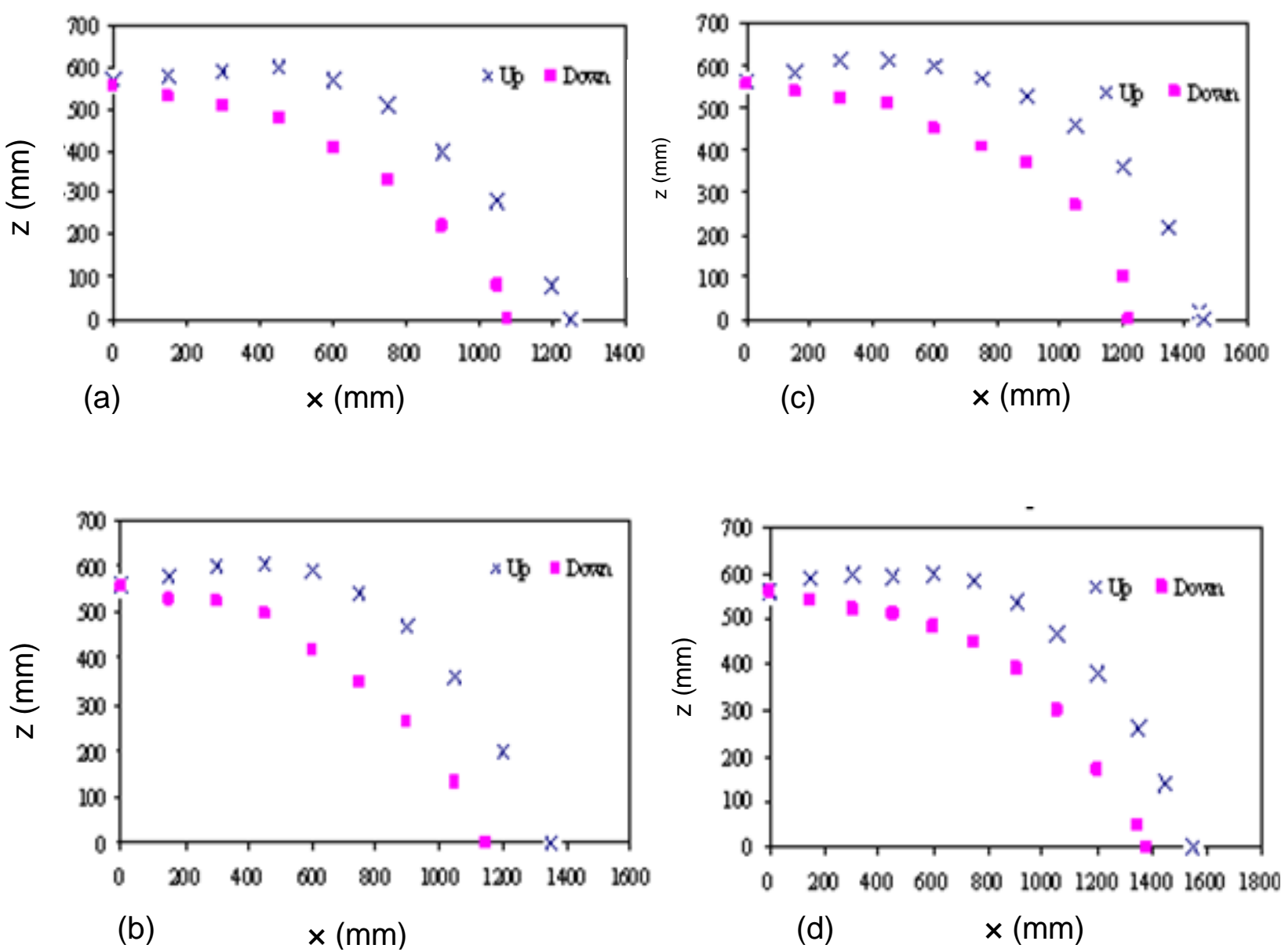

Figure 6. Limits of trajectory observed in experimental tests (a) $\theta_{C}=15^{\circ}$; (b) $\theta_{C}=30^{\circ}$; (c) $\theta_{C}=60^{\circ}$ and; (d) $\theta_{C}=90^{\circ}$.

results are shown for the upper limit of the trajectory. For instance, Figure $6 a$ shows this length equal to $1250 \mathrm{~mm}$ for $\theta_{c}=15^{\circ}$ and in Figure $6 \mathrm{~d}$, for $\theta_{c}=90^{\circ}$, it is almost $1575 \mathrm{~mm}$. This phenomenon is due to increasing of efflux momentum, which is reasonable. The momentum force is the major cause of jet power, which drive the jet flow forward. As a result, observation show that the convergence angle of jet nozzle significantly affects length trajectory. Table 3 presents the sample results corresponding to the change of upper limit coordinates.

In Table $3, Z_{0}$ is the highest elevation of the jet diameter, which is constant in all experiments, and $c$ variable is concentration of jet fluid, which plays role in the Densimetric Froude Number. In addition, increase of efflux velocity and Densimetric Froude Number lengthen the trajectory significantly. Table 4 demonstrate the changing of upper limit of trajectory to efflux velocity of jet flow.

\section{DISCUSSION}

Generally, on the base of experimental results which a sample of these results were presented in Table 3 , the comparison has shown increasing almost 25 to $30 \%$ of the development of trajectory, which has been due to changing of contraction angle from 15 to $90^{\circ}$. Also, as shown in the Table 4, increasing of efflux velocity up to 4 times is the cause of 10 times decreasing of $\left(Z_{0}-Z_{u}\right) / x_{u}$. $x_{u}$ is the $x$-coordinate of upper limit of trajectory. The main reason for the length of trajectory and contraction angle correlation is the increase of jet momentum flux, due to increase of contraction angle. The momentum flux is the main cause flow force, which is increased due to increases of contraction angle.

In this research, 96 types of neural networks, with different strategies (various threshold functions and various numbers of nods for hidden layers) for upper limit, and 96 types of neural networks with different strategies for inferior limit, were created. To achieve the goal, FFBP neural network was used. Two strategies were utilized for investigation of different threshold function effects on network optimization that include a one hidden layer and two hidden layers. Both strategies were used for FFBP network, with the learning algorithms of BP. The best results of the used network and algorithm 
Table 3. Statistics of contraction angle effects on the upper limit coordinates.

\begin{tabular}{|c|c|c|c|c|c|}
\hline \multicolumn{6}{|c|}{$\mathrm{dp}=8 \mathrm{~mm}, \mathrm{c}=30 \mathrm{~g} / \mathrm{L}, Q_{0}=0.026 \mathrm{~L} / \mathrm{s}$} \\
\hline \multicolumn{2}{|c|}{$A=15^{\circ}$} & \multicolumn{2}{|c|}{$A=45^{\circ}$} & \multicolumn{2}{|c|}{$A=90^{\circ}$} \\
\hline$(\mathrm{x} / \mathrm{dp}) \mathrm{u}$ & $(Z 0-Z u) / x u$ & $(\mathrm{x} / \mathrm{dp}) \mathrm{u}$ & $(Z 0-Z u) / x u$ & $(\mathrm{x} / \mathrm{dp}) \mathrm{u}$ & $(\mathrm{ZO}-\mathrm{Zu}) / \mathrm{xu}$ \\
\hline 0.00 & 0.00 & 0.00 & 0.00 & 0.00 & 0.00 \\
\hline 18.75 & -0.04 & 18.75 & -0.01 & 18.75 & -0.05 \\
\hline 37.50 & 0.09 & 37.50 & 0.28 & 37.50 & 0.06 \\
\hline 56.25 & 0.30 & 56.25 & 0.54 & 56.25 & 0.28 \\
\hline 75.00 & 0.67 & 75.00 & 0.91 & 75.00 & 0.49 \\
\hline 80.00 & 0.88 & 76.25 & 0.92 & 93.75 & 0.70 \\
\hline \multicolumn{6}{|c|}{$\mathrm{dp}=8 \mathrm{~mm}, \mathrm{c}=30 \mathrm{~g} / \mathrm{L}, Q_{0}=0.053 \mathrm{~L} / \mathrm{s}$} \\
\hline \multicolumn{2}{|c|}{$A=15^{\circ}$} & \multicolumn{2}{|c|}{$A=45^{\circ}$} & \multicolumn{2}{|c|}{$A=90^{\circ}$} \\
\hline$(\mathrm{x} / \mathrm{dp}) \mathrm{u}$ & $(Z 0-Z u) / x u$ & $(\mathrm{x} / \mathrm{dp}) \mathrm{u}$ & $(Z 0-Z u) / x u$ & $(\mathrm{x} / \mathrm{dp}) \mathrm{u}$ & $(\mathrm{ZO}-\mathrm{Zu}) / \mathrm{xu}$ \\
\hline 0.00 & 0.00 & 0.00 & 0.00 & 0.00 & 0.00 \\
\hline 18.75 & -0.11 & 18.75 & -0.11 & 18.75 & -0.11 \\
\hline 37.50 & -0.09 & 37.50 & -0.09 & 37.50 & -0.09 \\
\hline 56.25 & -0.01 & 56.25 & 0.01 & 56.25 & -0.06 \\
\hline 75.00 & 0.14 & 75.00 & 0.04 & 75.00 & 0.01 \\
\hline 93.75 & 0.26 & 93.75 & 0.17 & 93.75 & 0.09 \\
\hline 112.50 & 0.47 & 112.50 & 0.28 & 112.50 & 0.22 \\
\hline \multirow[t]{2}{*}{121.25} & 0.58 & 131.25 & 0.42 & 131.25 & 0.30 \\
\hline & & 141.25 & 0.50 & 150.00 & 0.47 \\
\hline
\end{tabular}

for upper and inferior limits are shown in Tables 5 and 6 .

As shown in Table 5, the best results of FFBP network in the first strategy for upper limit are related to 4-7-1 topology that produces RMSE $=0.0415, R^{2}=0.9774$ and $\mathrm{MAE}=0.005$, with train data. For test data, results were RMSE $=0.0452, R^{2}=0.9725$ and $\mathrm{MAE}=0.0052$. Furthermore, in this stage, application of SigmoidSigmoid has a best result, because it produced lower RMSE and MAE values and higher $R^{2}$ value.

As presented in Table 6 , the best results of FFBP network in the first strategy for inferior limit are related to 4-7-1 topology that produce RMSE $=0.0444, R^{2}=0.9766$ and $M A E=0.0045$, with train data. For test data result were RMSE $=0.0497, \mathrm{R}^{2}=0.9714$ and $\mathrm{MAE}=0.0051$. Furthermore, in this stage, application of Tangent Hyperbolic- Sigmoid has a best result, because it produced less RMSE and MAE values and more $R^{2}$ value.

The best results of FFBP network in the second strategy for up boundary are related to 4-7-7-1 topology that produce RMSE $=0.0401, \mathrm{R}^{2}=$ 0.9785 and $M A E=0.0045$, with train data. For test data, results were RMSE $=0.0435, R^{2}=0.9745$ and MAE $=$ 0.0048. Furthermore, in this stage, application of Sigmoid- Sigmoid has a best result, because it produced less RMSE and MAE values and more $R^{2}$ value.

The best results of FFBP network in the first strategy for down boundary are related to 4-7-7-1 topology that produce RMSE $=0.0382, \mathrm{R}^{2}=$ 0.9827 and $\mathrm{MAE}=0.0037$, with train data. For test data, results were RMSE $=0.0454, R^{2}=0.9762$ and $\mathrm{MAE}=$ 0.0044 . Furthermore, in this stage, application of Sigmoid- Sigmoid has a best result, because it produced less RMSE and MAE values and more $R^{2}$ value.

Figures 7 and 8 shows the relative length of jet's up and down boundaries versus pattern sequence of topology 4-7-7-1 for train and test data. The best $R^{2}$ for upper and inferior limits are shown in Figures 9 and 10, which belong to FFBP network, LM algorithm, and Sigmoid-Sigmoid-Sigmoid threshold functions, with topology 4-7-7-1 for both (up and down). In Figure 9 and 10 , predicted values of the jet trajectory were obtained from this network, compared to experimental results (real values). Sensitivity analysis of selected topologies resulted in the weight of each parameter in output value. This process is done by Qnet2000@ for upper and inferior limits separately. For upper boundary input values: relative length of jet trajectory, $\mathrm{X} / \mathrm{dp}$; jet convergence angel, $\theta_{c}$; geometry number of jet, Di/dp and; Densimetric Froude Number, $\mathrm{Fr}_{\mathrm{d}}$., have 38.07, 4.13, 30.6 and $27.2 \%$ effect on the output (relative height of jet trajectory Z/dp), respectively. For inferior limit input values; $X / d p, \theta_{c}$, Di/dp and $\mathrm{Fr}_{\mathrm{d}}$, have $33.98,4.5,34.76$ and $26.76 \%$ effect on the output (Z/dp), respectively.

\section{Conclusion}

In the present article, jet outfalls in the static ambient fluid have been physically simulated in the Hydraulic 
Table 4. Statistics of efflux velocity of the jet flow effects on the upper limit coordinates.

\begin{tabular}{|c|c|c|c|c|c|c|c|}
\hline \multicolumn{8}{|c|}{ Angle $=15^{\circ}, \mathrm{dp}=5 \mathrm{~mm}, \mathrm{C}=15 \mathrm{~g} / \mathrm{L}$} \\
\hline \multicolumn{2}{|c|}{$\mathrm{Fr}=34.91 ; \mathrm{U}_{0}=0.5 \mathrm{~m} / \mathrm{s}$} & \multicolumn{2}{|c|}{$\mathrm{Fr}=57.36 ; \mathrm{U}_{0}=1.0 \mathrm{~m} / \mathrm{s}$} & \multicolumn{2}{|c|}{$\mathrm{Fr}=89.78 ; \mathrm{U}_{0}=1.5 \mathrm{~m} / \mathrm{s}$} & \multicolumn{2}{|c|}{$\mathrm{Fr}=119.71 ; \mathrm{U}_{0}=2.0 \mathrm{~m} / \mathrm{s}$} \\
\hline$(x / d p) u$ & $\left(Z_{0}-Z u\right) / x u$ & $(x / d p) u$ & $\left(Z_{0}-Z u\right) / x u$ & $(x / d p) u$ & $\left(Z_{0}-Z u\right) / x u$ & $(x / d p) u$ & $\left(Z_{0}-Z u\right) / x u$ \\
\hline 0.00 & 0.00 & 0 & 0 & 0 & 0 & 0 & 0 \\
\hline 30.00 & -0.18 & 30 & -0.083 & 30 & -0.183 & 30 & -0.117 \\
\hline 60.00 & -0.06 & 60 & -0.025 & 60 & -0.125 & 60 & -0.092 \\
\hline 90.00 & 0.11 & 90 & 0.006 & 90 & -0.083 & 90 & -0.083 \\
\hline 120.00 & 0.39 & 120 & 0.138 & 120 & -0.0459 & 120 & -0.071 \\
\hline \multirow[t]{8}{*}{140.00} & 0.80 & 150 & 0.217 & 150 & 0.01 & 150 & -0.063 \\
\hline & & 180 & 0.325 & 180 & 0.081 & 180 & -0.042 \\
\hline & & 210 & 0.45 & 210 & 0.14 & 210 & -0.036 \\
\hline & & 214 & 0.526 & 240 & 0.194 & 240 & 0.018 \\
\hline & & & & 270 & 0.283 & 270 & 0.076 \\
\hline & & & & 290 & 0.388 & 290 & 0.133 \\
\hline & & & & & & 320 & 0.214 \\
\hline & & & & & & 340 & 0.331 \\
\hline \multicolumn{8}{|c|}{ Angle $=15^{\circ} \mathrm{dp}=5 \mathrm{~mm} \mathrm{C}=50 \mathrm{~g} / \mathrm{L}$} \\
\hline \multicolumn{2}{|c|}{$\mathrm{Fr}=16.68 ; \mathrm{U}_{0}=0.5 \mathrm{~m} / \mathrm{s}$} & \multicolumn{2}{|c|}{$\mathrm{Fr}=33.36 ; \mathrm{U}_{0}=1.0 \mathrm{~m} / \mathrm{s}$} & \multicolumn{2}{|c|}{$\mathrm{Fr}=47.47 ; \mathrm{U}_{0}=1.5 \mathrm{~m} / \mathrm{s}$} & \multicolumn{2}{|c|}{$\mathrm{Fr}=61.59 ; \mathrm{U}_{0}=2.0 \mathrm{~m} / \mathrm{s}$} \\
\hline$(x / d p) u$ & $\left(Z_{0}-Z u\right) / x u$ & $(x / d p) u$ & $\left(Z_{0}-Z u\right) / x u$ & $(x / d p) u$ & $\left(Z_{0}-Z u\right) / x u$ & $(x / d p) u$ & $\left(Z_{0}-Z u\right) / x u$ \\
\hline 0.00 & 0.00 & 0 & 0 & 0 & 0 & 0 & 0 \\
\hline 30.00 & -0.11 & 30 & -0.116 & 30 & -0.09 & 30 & -0.05 \\
\hline 60.00 & 0.18 & 60 & 0.008 & 60 & -0.072 & 60 & -0.092 \\
\hline 90.00 & 0.78 & 90 & 0.161 & 90 & -0.0056 & 90 & -0.028 \\
\hline \multirow[t]{5}{*}{98.00} & 1.15 & 120 & 0.388 & 120 & 0.138 & 120 & 0.088 \\
\hline & & 148 & 0.760 & 150 & 0.256 & 150 & 0.163 \\
\hline & & & & 180 & 0.458 & 180 & 0.303 \\
\hline & & & & 200 & 0.562 & 210 & 0.489 \\
\hline & & & & & & 214 & 0.526 \\
\hline
\end{tabular}

Table 5. Strategies for different neurons and hidden layers for several topologies in the training and test steps, upper limit.

\begin{tabular}{|c|c|c|c|c|c|c|c|c|c|}
\hline \multirow{3}{*}{$\begin{array}{l}\text { No. of } \\
\text { hidden } \\
\text { layer }\end{array}$} & \multirow{3}{*}{$\begin{array}{c}\text { No. of } \\
\text { hidden } \\
\text { layers } \\
\text { node }\end{array}$} & \multirow{2}{*}{\multicolumn{2}{|c|}{$\begin{array}{l}\text { Upper boundary threshold } \\
\text { function }\end{array}$}} & \multicolumn{6}{|c|}{ Assessment criteria } \\
\hline & & & & \multicolumn{2}{|c|}{$\mathbf{R}^{2}$} & \multicolumn{2}{|c|}{ RMSE } & \multicolumn{2}{|c|}{ MAE } \\
\hline & & Hidden layers & Output layers & Train & Test & Train & Test & Train & Test \\
\hline 1 & 3 & $\begin{array}{l}\text { Tangent } \\
\text { hyperbolic }\end{array}$ & $\begin{array}{l}\text { Secant } \\
\text { hyperbolic }\end{array}$ & 0.9671 & 0.9655 & 0.0496 & 0.0511 & 0.0064 & 0.0067 \\
\hline 1 & 5 & Sigmoid & Sigmoid & 0.9722 & 0.9692 & 0.0457 & 0.0483 & 0.0056 & 0.0061 \\
\hline 1 & 7 & Sigmoid & Sigmoid & 0.9774 & 0.9725 & 0.0415 & 0.0452 & 0.005 & 0.0052 \\
\hline 2 & 3,3 & $\begin{array}{l}\text { Tangent } \\
\text { hyperbolic }\end{array}$ & Sigmoid & 0.969 & 0.9689 & 0.048 & 0.0481 & 0.006 & 0.0059 \\
\hline 2 & 5,5 & $\begin{array}{l}\text { Tangent } \\
\text { hyperbolic }\end{array}$ & Sigmoid & 0.9773 & 0.9718 & 0.0412 & 0.0461 & 0.0047 & 0.0052 \\
\hline 2 & 7,7 & Sigmoid & Sigmoid & 0.9785 & 0.9745 & 0.0401 & 0.0435 & 0.0045 & 0.0048 \\
\hline
\end{tabular}

Laboratory of Shahid Chamran University, Iran. A large amount of data has been collected from 192 runs of physical models. Then, gathered observation data has been used for mathematical simulation using Artificial 
Table 6. Strategies for different neurons and hidden layers for several topologies in the training and test steps, inferior limit.

\begin{tabular}{|c|c|c|c|c|c|c|c|c|c|}
\hline \multirow{3}{*}{$\begin{array}{l}\text { No. of } \\
\text { hidden } \\
\text { layer }\end{array}$} & \multirow{3}{*}{$\begin{array}{c}\text { No. of } \\
\text { hidden } \\
\text { layers } \\
\text { node }\end{array}$} & \multirow{2}{*}{\multicolumn{2}{|c|}{$\begin{array}{l}\text { Upper boundary threshold } \\
\text { function }\end{array}$}} & \multicolumn{6}{|c|}{ Assessment criteria } \\
\hline & & & & \multicolumn{2}{|c|}{$\mathbf{R}^{2}$} & \multicolumn{2}{|c|}{ RMSE } & \multicolumn{2}{|c|}{ MAE } \\
\hline & & Hidden layers & Output layers & Train & Test & Train & Test & Train & Test \\
\hline 1 & 3 & Sigmoid & $\begin{array}{l}\text { Secant } \\
\text { hyperbolic }\end{array}$ & 0.9681 & 0.965 & 0.0518 & 0.0551 & 0.0056 & 0.0061 \\
\hline 1 & 5 & $\begin{array}{l}\text { Tangent } \\
\text { hyperbolic }\end{array}$ & Sigmoid & 0.9748 & 0.971 & 0.0468 & 0.0501 & 0.0047 & 0.0052 \\
\hline 1 & 7 & $\begin{array}{l}\text { Tangent } \\
\text { hyperbolic }\end{array}$ & Sigmoid & 0.9766 & 0.9714 & 0.0444 & 0.0497 & 0.0045 & 0.0051 \\
\hline 2 & 3. 3 & Sigmoid & $\begin{array}{l}\text { Secant } \\
\text { hyperbolic }\end{array}$ & 0.9649 & 0.971 & 0.046 & 0.05 & 0.0046 & 0.0051 \\
\hline 2 & 5.5 & Sigmoid & Sigmoid & 0.978 & 0.9742 & 0.043 & 0.0472 & 0.0042 & 0.0046 \\
\hline 2 & 7.7 & Sigmoid & Sigmoid & 0.9727 & 0.9762 & 0.0382 & 0.0454 & 0.0037 & 0.0044 \\
\hline
\end{tabular}
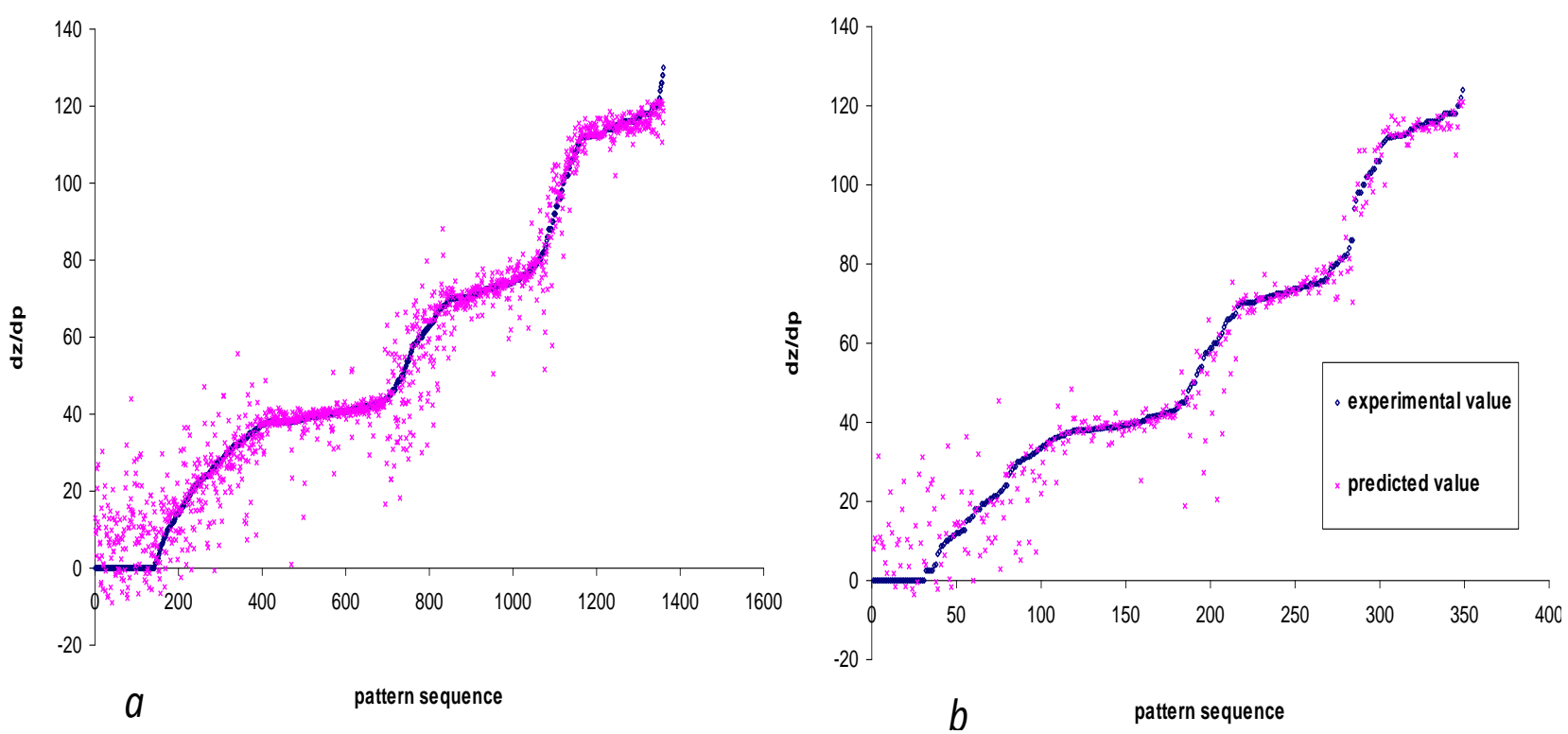

Figure 7. Relative length of jet's upper boundary and pattern sequence for topology 4-7-7-1,(a) Train data and (b) test data (the $x$ axis denotes row number of arranged data from low high values of $z / d p$ ).

Neural Networks (ANN). Observed data showed that increase in contraction angle of jet is the main caused for increasing the length of upper and lower limits of the jet trajectory.

Among the ANNs networks, including FFBP network, BP algorithm, and Sigmoid-Sigmoid-Sigmoid threshold functions, with seven neurons for the first hidden layer, and seven neurons for the second hidden layer, were employed in the simulation process. Utilizing more than two hidden layers and seven nodes in hidden layers, in most of topologies, caused fluctuations and lack of stability, and sometimes divergence of neural networks. Neural network training parameters, such as learning rate and momentum factor, based on software recommendations and trial and error, found 0.01 and 0.8 , respectively that causes minimal error and stability of training process. Sensitivity analysis of influence of the input parameters on output parameters showed that $\mathrm{x} / \mathrm{dp}$ and $\mathrm{Di} / \mathrm{dp}$ had the highest influence on $\mathrm{z} / \mathrm{dp}$.

Finally, however, several known and unknown 


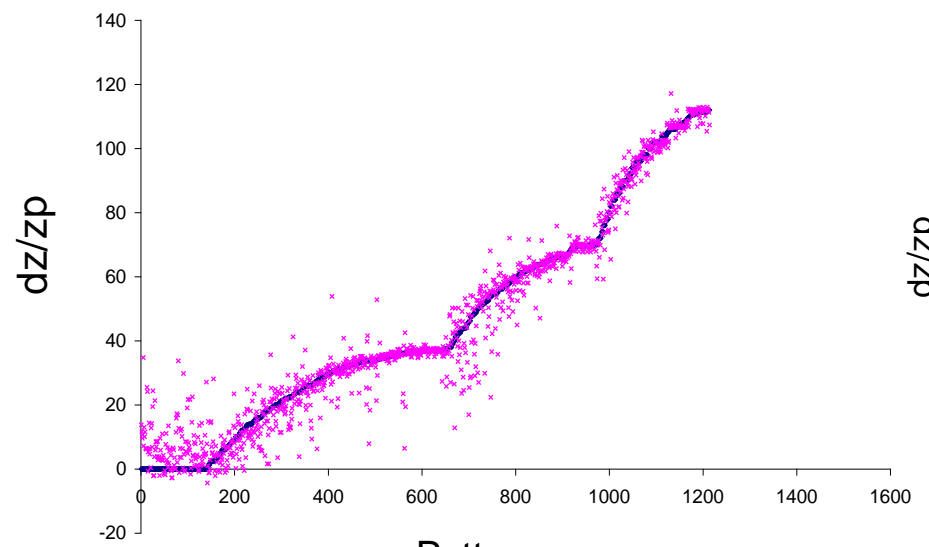

(a)
Pattern sequence

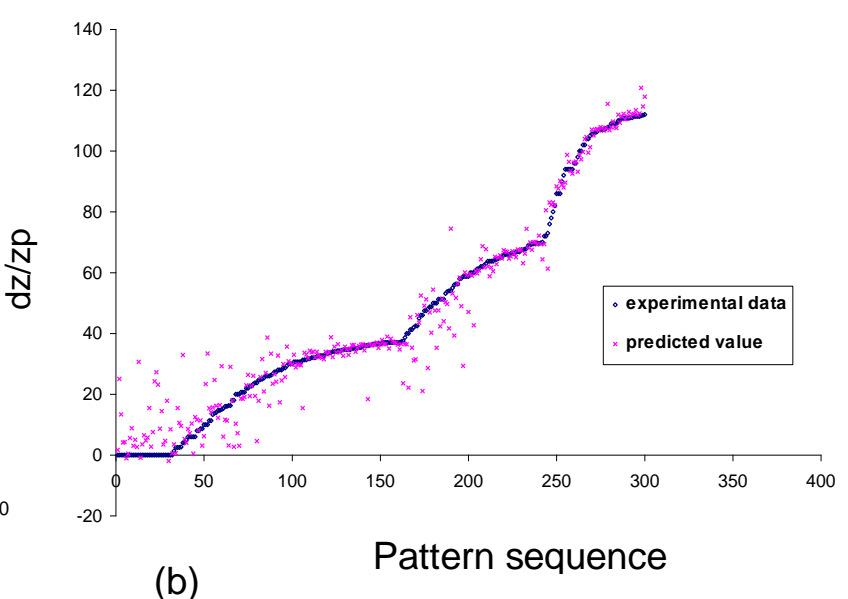

(b)

Figure 8. Relative length of jet's lower boundary and pattern sequence for topology 4-7-7-1. (a) Train data and (b) test data (the $x$ axis denotes row number of arrange data from low high values of $\mathrm{z} / \mathrm{dp}$ ).

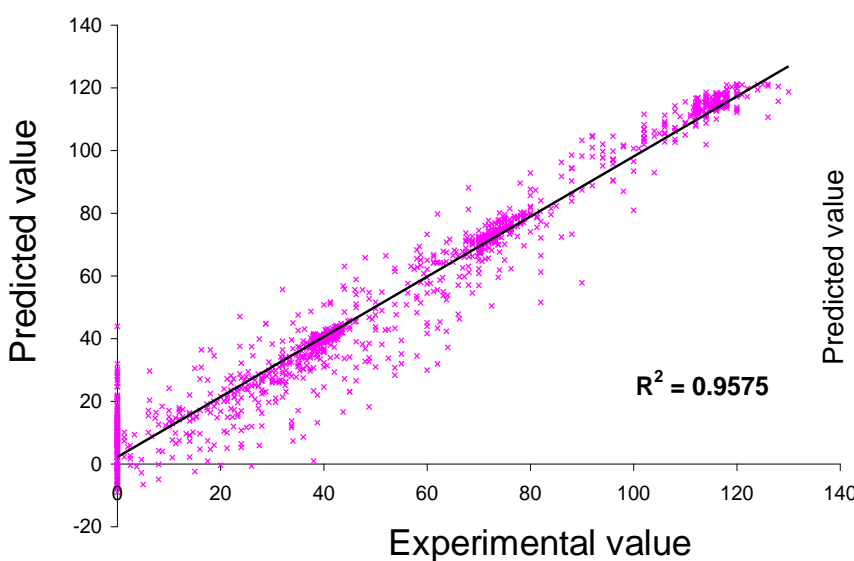

(a)

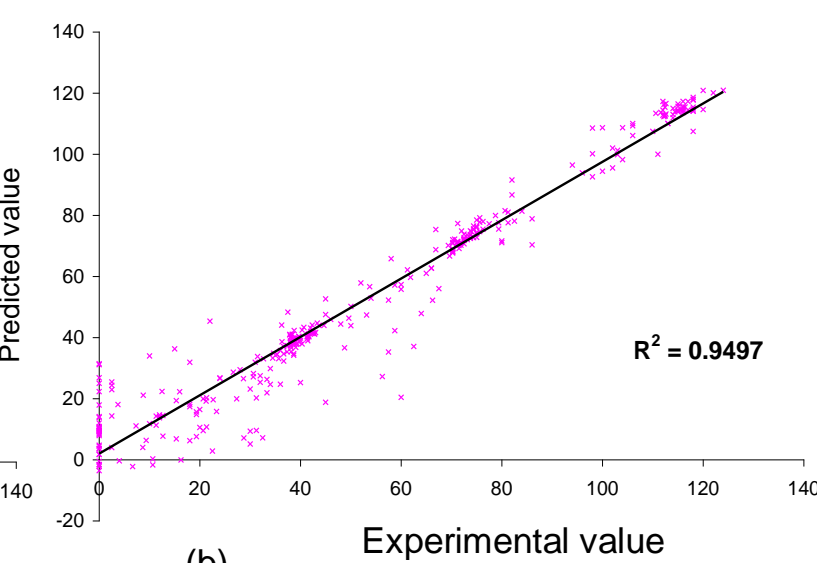

(b)

Figure 9. Predicted values of jet's upper boundary using optimum network versus experimental values and determination coefficient of optimum network (a) training step (b) test step.

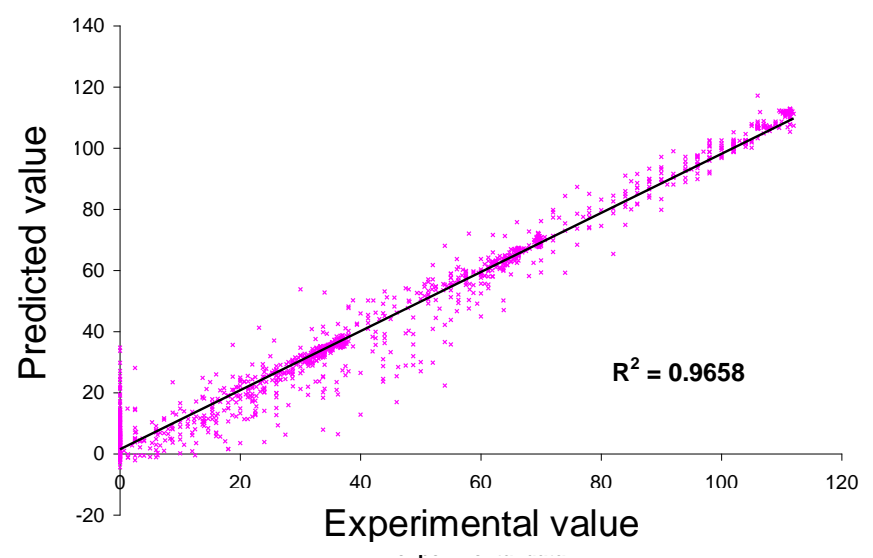

(a)

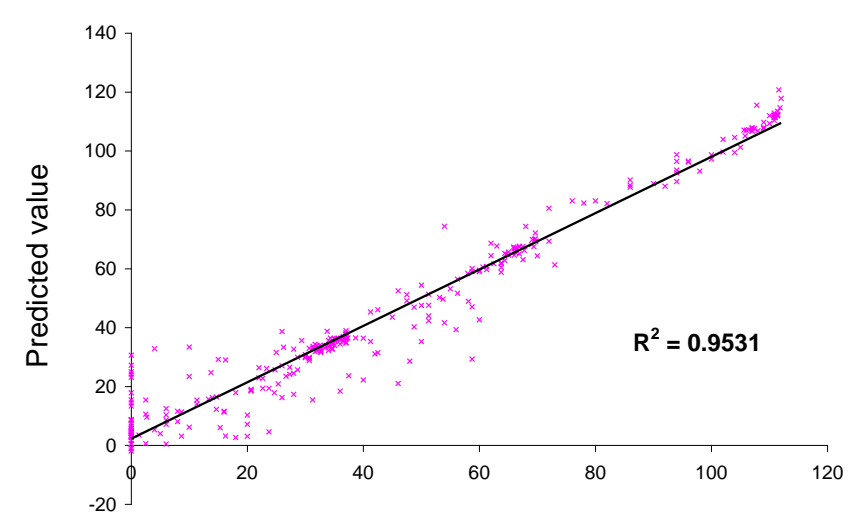

(b)

\section{Experimental value}

Figure 10. Predicted values of jets down boundary using optimum network versus experimental values and determination coefficient of optimum network (a) training step (b) test step. 
parameters affect the jet outfalls in the stagnant ambient fluid; ANN is able to simulate jet outfalls characteristics very successfully.

\section{ACKNOWLEDGEMENTS}

The authors would like to acknowledge Shahid Chamran University of Ahwaz and the Centre of Excellence on Operation Management of Irrigation and Drainage Networks for financial support and facilitation of the experiments. The financial support of Khuzestan Province Water and Sewage Organization is appreciated.

\section{REFERENCES}

Adineh VR, Aghanajafi C, Dehghan GH, Jelvani S (2008). Optimization of the operational parameters in a fast axial flow CW CO2 laser using artificial neural networks and genetic algorithms. Opt. Laser Technol. 40:1000-1007.

Adrian WL, Stephan GM (2004). Double diffusive effect on desalination discharges. J. Hyd. Eng. 122:450-457.

Ahadiyan J, Musavi-Jahrom SH (2008). Investigation of variation of efflux momentum in shallow receiving water using FLOW-3D. Proceeding of International Symposium of Water Resource Management Tabriz, Iran. pp. 551-557.

Albertson ML, Dai YB, Jenson RA, Rouse H (1950). Diffusion of submerged jets. Trans. Am. Soc. Civil Eng. 115:639-664.

Ben M, Davies P, Malcangio D, Mossa M, Petrillo AF (2004). Turbulence of vertical round buoyant jets in a cross flow. In: Greco Carravetta, Della Morte (eds) River Flow 2004: Proc. An International workshop held at Napoli, Italy. London: AA Balkema, 2:1167-1174.

Cuthberston JS, Peter A, Davis PA (2008). Deposition from particleladen round turbulent horizontal buoyant jets in stationary and coflowing receiving fluids. J. Hyd. Eng. 134:390-402.
Demuth H, Beale M, Hagan M (2007). Neural network toolbox 5 user's guide. The Mathworks Inc.

Fischer HB, List EJ, Koh RCY, Imberger J, Brooks NH (1979). Mixing in inland and coastal waters. Academic Press.

French RH (1986). Open channel hydraulics. McGraw-Hill Book Company. pp. 509-548.

Gallant SI (1993). Neural network learning and expert systems. Cambridge: MIT Press.

Girosi F, Jones M, Poggio T (1995). Regularization theory and neural network architectures. Neural Comp. 7(2):19-69.

Hagan MT, Menhaj MB (1994). Training feed forward networks with the Marquardt algorithm. IEEE Trans Neural Netw. 5:989-993.

Kasabov NK (1998). Foundations of neural networks fuzzy systems and knowledge engineering. Cambridge: MIT Press.

Kozma R, Sakuma M, Yokoyama Y, Kitamura M (1996). On the accuracy of mapping back propagation with forgetting. Neurocomp. 13:295-311.

Kunze E (1987). Limits on growing, finite-length fingers: A Richardson number constraint. J. Mar. Res. 45:533-556.

Lee JHW, Chu V H (2003). Turbulent Jets and plumes- a lagrangian approach. Kluwer Academic Publishers. Boston.

Maxworthy T (1983). The dynamics of double diffusive gravity currents. J. Fluid Mech. 128:259-282.

Pantokratoras A (2003). Vertical penetration of double diffusive water plumes discharged vertically downward. J. Hyd. Eng. 129:541-545.

Papanicolau PN, List EJ (1988). Investigation of round vertical turbulent buoyant jets. J. Fluid Mech. 195:341-391.

Pirmoradian N, Safshekan F, Sharifan AR (2009). Simulating hydrograph's peak time of occurrence using artificial neural networks in kasilian represent basin. ICWR 2009 International Conference, Shahrood, Iran.

Rajaratnam N (1976). Turbulent jets. Elsevier Scientific Publishing Company.

Turner JS (1998). Stratification and circulation produced by heating and evaporation on a shelf. J. Mar. Res. 56:855-904.

Turner JS (1967). Salt fingers a density interface. Deep-sea Res. Oceanogr. Abstr. 14:599-611. 